Revue de droit comparé du travail et de la sécurité sociale

$2 \mid 2020$

La vie personnelle du salarié

\title{
Vie personnelle et relation de travail dans la jurisprudence en Turquie
}

Melda Sur

\section{(2) OpenEdition}

1 Journals

Édition électronique

URL : https://journals.openedition.org/rdctss/1078

DOI : $10.4000 /$ rdctss. 1078

ISSN : 2262-9815

Éditeur

Centre de droit comparé du travail et de la sécurité sociale

Édition imprimée

Date de publication : 1 juin 2020

Pagination : 56-65

ISSN : 2117-4350

\section{Référence électronique}

Melda Sur, « Vie personnelle et relation de travail dans la jurisprudence en Turquie », Revue de droit

comparé du travail et de la sécurité sociale [En ligne], 2 | 2020, mis en ligne le 01 novembre 2021, consulté le 11 novembre 2021. URL : http://journals.openedition.org/rdctss/1078 ; DOI : https:// doi.org/10.4000/rdctss. 1078

\section{(c) $(7)(9$}

Revue de droit comparé du travail et de la sécurité sociale est mise à disposition selon les termes de la Licence Creative Commons Attribution - Pas d'Utilisation Commerciale - Pas de Modification 4.0 International. 


\section{VIE PERSONNELLE ET RELATION DE TRAVAIL DANS LA JURISPRUDENCE EN TURQUIE}

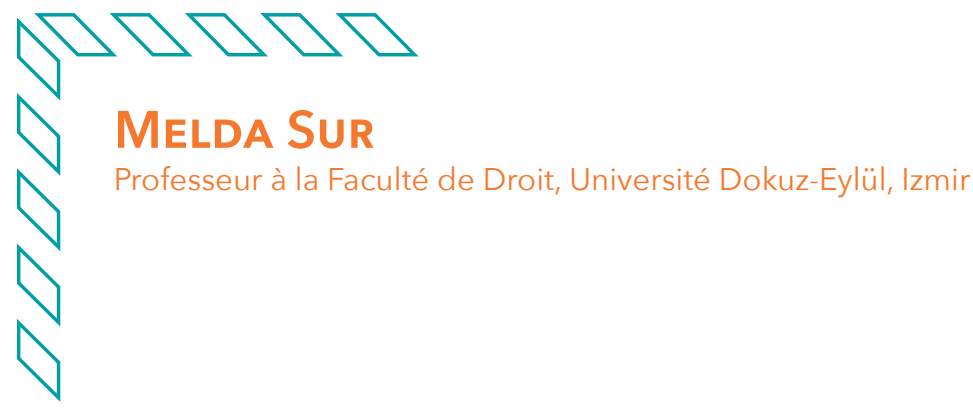

\section{RÉSUMÉ}

En Turquie, le droit au respect de la vie privée et la protection des données personnelles sont garantis par la Constitution et la législation. Le juge s'efforce de concilier et de maintenir un équilibre entre, d'une part les nécessités liées à la bonne marche de l'entreprise, et, d'autre part, les droits fondamentaux des travailleurs, et plus particulièrement la liberté de choix de vie des salariés. Une jurisprudence s'est façonnée à partir de certains aspects de la vie personnelle, comme les saisies répétées ou l'usage de boissons alcoolisées, causes de rupture dans la mesure où ils ont un impact sur le travail. Les relations « inappropriées » entre salariés peuvent être un motif de licenciement si elles ont des incidences négatives sur le milieu de travail. L'usage abusif de l'internet dans l'entreprise peut être dépisté et les messages captés admis comme moyen de preuve. Toutefois, la jurisprudence semble évoluer vers une certaine protection contre la surveillance de l'internet et le captage des messages, en exigeant désormais que les salariés en soient préalablement informés.

Mots-clés : Vie privée, usage de l'internet, données personnelles, rupture du contrat de travail, droits fondamentaux au travail

\footnotetext{
ABSTRACT

In Turkey the rights to the protection of private life and personal data have been guaranteed by the Constitution and the law. Courts tend to conciliate and maintain a just balance between the needs and requirements of the enterprise and the fundamental rights of the workers, especially on issues regarding their choice of life. A case law has emerged on some aspects of personal life, such as recurrent debts and the use of alcoholic drinks, considered as causes of termination when they have adverse effects on labor. Non appropriate relations among colleagues may cause termination if they had negative effects at the workplace. On computers and internet provided by the management, the use for personal purposes and emails may be monitored and records kept by the management, and Courts traditionally accept such records as means of proof. However, the case law on this issue seems to evolve towards a more restrictive approach, and in recent decisions the higher Courts tend to require that the management previously inform their employees of such intrusion and monitoring.

Key words: Private Life, Use of the Internet, Personal Data Protection, Termination of Employment, Fundamental Rights of the Worker
} 


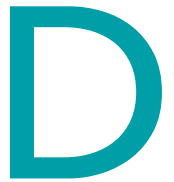

ans quelle mesure la vie personnelle du salarié peut-elle influer sur sa vie au travail ? Notre étude jurisprudentielle portera sur la prise en compte de la « vie privée » du salarié et sa protection dans les relations de travail, sans toutefois entrer dans le vaste domaine de la conciliation du travail avec les contraintes familiales qui, faisant l'objet d'une législation complexe, mériterait un examen à part.

Le droit au respect de la vie privée est protégé dans divers textes internationaux auxquels la Turquie a été partie, notamment par la Convention Européenne des Droits de l'Homme ${ }^{1}$ dont le système de protection juridictionnelle a une forte influence sur la jurisprudence turque et en particulier sur celle de la Cour Constitutionnelle. L'article 20 de la Constitution turque, intitulé "secret de la vie privée », énonce que toute personne a droit au respect de sa vie privée et familiale, et que le secret de la vie privée doit être protégé (alinéa ${ }^{\text {r}}$ ). Cette disposition, sujette à des exceptions d'ordre public, est suivie par un troisième alinéa plus récemment ajouté portant sur la protection des données personnelles.

Or, la notion de vie privée n'est pas toujours aisée à définir. Le Code Civil turc protège les «droits de la personne " à l'article 24 de manière assez large, comprenant l'intégrité physique et des valeurs telles que la vie privée, l'honneur, le nom, l'image, les libertés, les secrets et croyances, qui entrent dans son domaine d'application.

Dans ce contexte juridique, qu'en est-il du monde du travail où le salarié est soumis à un lien de subordination envers l'employeur, se trouve parfois confronté à des traitements discriminatoires ou perd son emploi pour des raisons touchant à sa vie privée ? Le sujet a fait l'objet de thèses et d'études si bien qu'une jurisprudence s'est formée.

Il convient donc d'examiner les arrêts qui se sont façonnés à l'occasion de licenciements, en commençant par la vie privée du salarié en dehors du lieu de travail (I), puis sur le lieu de travail, en mettant l'accent sur les problèmes actuels liés à l'usage des moyens informatiques par le salarié et à leur dépistage par la direction (II).

\section{I - LA VIE PERSONNELLE EN DEHORS DE L'ENTREPRISE}

À priori, en dehors de l'entreprise, chaque personne devrait pouvoir mener sa vie comme elle l'entend. Or on voit que dans certains cas, des antécédents ou des évènements ayant eu lieu avant l'embauche peuvent avoir une incidence sur la relation de travail et sa rupture (A).

De même, après que le travailleur se voie embauché, il arrive que des agissements en dehors de la sphère du travail soient considérés comme ayant des effets négatifs sur la relation de travail (B). 


\section{A - LA VIE PRIVÉE LORS DE L'EMBAUCHE ET LA SAUVEGARDE D'INFORMATIONS ACQUISES}

On admet dans la doctrine qu'il existe une obligation de protection dès la conclusion du contrat de travail, obligation dont le non-respect lors des entretiens d'embauche constituerait une faute culpa in contrahendo qui donnerait droit à des dommages et intérêts. L'obligation de respecter la vie privée du futur salarié commence donc préalablement à l'embauche'2.

Ainsi, lors des entretiens, le futur salarié peut ne pas répondre à certaines questions sans rapport ou incidence sur l'exécution du travail. En revanche, il est tenu de révéler des faits qui auraient un impact sur l'exécution du travail. Les exemples jurisprudentiels les plus pertinents concernent la non révélation d'antécédents de condamnations pénales de personnes recrutées dans des services de sécurité. Dans certains cas examinés, la case relative à « l'existence de condamnations antérieures » figurant au formulaire d'embauche n'avait pas été cochée, et par la suite la rupture du contrat fut considérée comme justifiée ${ }^{3}$. Cette jurisprudence est bien établie, mais certains arrêts ne semblent toutefois pas faire de distinction entre le type de condamnation et la nature du poste à occuper ${ }^{4}$.

Quant à la sauvegarde et à l'usage des informations recueillies par la direction, le Code turc des Obligations souligne que l'employeur est tenu de protéger et respecter la personnalité du travailleur ${ }^{5}$ et qu'il « fera usage des données personnelles de son salarié seulement si celles-ci sont relatives à son aptitude au travail ou dans la mesure où elles seraient nécessaires à l'exécution du contrat de travail. Les dispositions des lois spéciales sont réservées $»^{6}$.

Enfin, le même article prévoit que «l'usage des données et renseignements acquis par l'employeur sur son salarié doit s'exercer de manière conforme au principe de bonne foi et de manière légale, et les renseignements que le salarié a un intérêt légitime à garder secrets ne devraient pas être divulgués ${ }^{7}$.

2 Ş. Ertürk, İ̧̧ Ilişkisinde Temel Haklar, Ankara 2002, p. 63; A. Sevimli, Iş̧̧̧inin Özel Yaşamına Müdahalenin Sınırları, İstanbul 2006, p. 145 ; B. Erkanlı, Tarafların İ̧̧ Sözleşmesi Yapılması Sırasındaki Hak ve Yükümlülükleri, Ankara 2015, p. 62 ; E.S. Gürkan, «Türk İş Hukukunda İşverenin İşe Alım Görüşmelerinde Aday İşçiye Soru Sorma Hakkının Sınırları », İstanbul Maltepe Üniversitesi Hukuk Fakültesi, 2017, n¹-2, p. 111.

$39^{e}$ Chambre civile de la Cour de Cassation, 22.1.1998, 19393/432, Tekstil İşveren, Mai-Juin 1998, p. 15. Dans le même sens : 9 Chambre civile de la Cour de Cassation, 11.11.1981, 19728/13806, Günay, İş Kanunu Şerhi, Ankara 2015, p. 1029.

$49^{e}$ Chambre civile de la Cour de Cassation, 6.6.2000, 4727/7993, Çimento İşveren, $n^{\circ} 4$, vol.14, Juillet 2000, p. 44.

5 Art. 417.

6 Art. 419.

7 Sur le sujet de la protection des données personnelles, voir notamment i. Gürsel, İşçinin Kişisel Verilerinin Korunması Hakkı, Ankara, 2016; I Gürsel, Kişisel Verilerin Korunması Hakkının İşçi ve Işveren iliş̧kisine Etkisi, Legal IHSGHD, n50, 2016, p. 763. 


\section{B - L'INCIDENCE DE LA VIE PRIVÉE SUR LA RELATION DE TRAVAIL}

\section{1 - Approche générale}

L'article 18 du Code du Travail (İş Kanunu) régit les causes valables de licenciement, et son exposé des motifs précise « qu'un agissement socialement répréhensible du travailleur, un comportement qui n'est point approuvé du point de vue social et éthique, mais qui n'a aucun effet négatif sur la production et la relation de travail, ne saurait constituer un motif valable de licenciement ».

Ainsi est-il nécessaire pour qu'un licenciement soit considéré comme valable, que le comportement du salarié ait affecté la prestation de service et la relation de travail, de manière à véritablement justifier une rupture.

Le Code du Travail distingue deux sortes de licenciement : ceux essentiellement dus à une faute grave du salarié ${ }^{8}$ où ni préavis ni indemnités ne sont dus, et les ruptures " pour causes valables ${ }^{9}$ de moindre gravité, aux conséquences moins défavorables, qui donnent lieu à préavis et indemnités. II appartient au juge de décider s'il y a disproportion ou non entre l'agissement reproché et la rupture ou type de rupture, considérée comme une mesure admissible en dernier ressort (ultima ratio). Par conséquent, seuls des comportements et une vie privée entrainant véritablement des incidences sur le travail peuvent justifier un licenciement.

La jurisprudence s'est développée sur plusieurs axes : I'usage de l'alcool, les endettements et saisies, l'expression d'opinions, la vie sentimentale du salarié et les relations entre collègues.

\section{2 - Le mode de vie du salarié}

Il ne saurait être question d'exiger que le mode de vie des salariés en dehors de l'établissement soit conforme aux valeurs préconisées par l'employeur. Toutefois, des exceptions peuvent être admises liées aux intérêts et à l'image de l'entreprise. Ainsi, un haut responsable qui représente l'établissement devrait prendre un certain soin de sa vie privée, dans le cadre du devoir de fidélité dû à l'employeur ${ }^{10}$.

Par ailleurs, le Code du Travail prévoit parmi les causes justifiées de licenciement, un « arrêt de travail excédant trois jours de travail consécutifs, ou cinq jours de travail en un mois, dû à une maladie ou invalidité sciemment causée ou résultant d'une vie désordonnée ou de l'abus d'alcool ». Cette juste cause de licenciement donne néanmoins droit à I'indemnité de rupture ${ }^{11}$. Les activités professionnelles en dehors des heures de travail sont tolérées, à condition que le salarié n'entre pas en concurrence avec l'employeur. De même, ces activités ne devraient pas nuire au rendement du travail.

8 Art. 25.

9 Art. 18.

10 Sevimli, p. 243.

11 Art. 25/l a du Code du Travail n4857 et art. 14 du Code du Travail n¹475. 
Le législateur en Turquie montre traditionnellement une certaine méfiance à l'égard de l'usage de boissons alcoolisées. Actuellement c'est l'article 28/1 de la Loi n6331 sur la Santé et la sécurité du travail ${ }^{12}$ qui prohibe, comme l'avait fait auparavant le Code du Travail, l'usage de boissons alcoolisées et de drogues dans l'établissement; par ailleurs estil interdit au salarié de se présenter à l'établissement en état d'ébriété ou ayant consommé une drogue. Le non-respect de ces dispositions constitue une faute grave cause de licenciement ${ }^{13}$.

La traditionnelle interdiction concernant les boissons alcoolisées fait néanmoins l'objet d'une jurisprudence assez mesurée. Selon la Cour de Cassation, si le salarié n'est pas ivre, le seul fait d'avoir bu avant de se présenter au travail ne peut être une juste cause de rupture ${ }^{14}$. Comme la Cour le souligne pertinemment, « si la quantité d'alcool ingérée (hors du lieu de travail) n'a pas eu d'effets sur la volonté et les comportements ou le travail, le seul fait d'avoir fait usage de boissons alcoolisées en dehors du lieu de travail ne peut constituer un motif suffisant de rupture $»^{15}$.

Quant à l'endettement du salarié, les tribunaux admettent dans des cas extrêmes qu'un salarié puisse se voir licencié pour cause d'endettement et saisies répétées portées sur son salaire, lorsque ces poursuites nuisent à la bonne marche de l'entreprise.

Dans certains cas, la Cour de Cassation a relevé que les services comptables de l'entreprise auraient été accaparés par ces saisies successives, au point de subir une surcharge de travail ${ }^{16}$. Encore est-il nécessaire de prévenir le salarié ${ }^{17}$. En revanche, dans le cas où le travailleur a contracté ses dettes quand il a été licencié et s'est trouvé sans emploi, la $22^{\mathrm{e}}$ Chambre de la Cour de Cassation a considéré la rupture pour dettes comme injustifiée ${ }^{18}$.

12 iş̧ Sağlığı ve Güvenliği Kanunu, Journal Officiel du 30.6.2012, n²8339.

13 Art. 25/II d.

$149^{e}$ Chambre civile de la Cour de Cassation, 8.5.2003, 25918/7770, Kazancı. Dans le même sens, voir $9^{e}$ Chambre civile de la Cour de Cassation, 9.10.2002, 4239/18575; $9^{e}$ Chambre civile de la Cour de Cassation, 27.6.1978, 6424/9324, Tekstil İşveren, n³4, Janvier 1979, p. 9.

$159^{e}$ Chambre civile de la Cour de Cassation, 27.6.1978, 6424/9324, Tekstil İşveren, n³4, Janvier 1979, p. 9.

$169^{e}$ Chambre civile, 2.2.2009, 9773/914, Kar, İş Güvencesi, p. 526 ; $9^{\mathrm{e}}$ Chambre civile, 24.3.2008, 10363/6019. Dans ses arrêts, la Cour de Cassation fait souvent mention d'une « tendance habituelle du salarié à l'endettement et au non payement de ses dettes »: 9e Chambre civile, 9.2.2009, 13284/1753, Çil, iş Hukuku Yargıtay Illke Kararları, p. 294 ; 9 e Chambre civile, 2.2.2009, 9800/1012, Legal 2009, n²2, p. 789.

$179^{e}$ Chambre civile, 24.3.2008, 10363/6019, Legal 2008, nº18, p. 674.

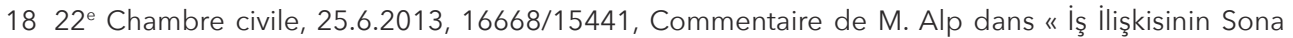
Ermesi ve Kıdem Tazminatı ", Yargıtay'ın Iş Hukuku ve Sosyal Güvenlik Hukuku Kararlarının Değerlendirilmesi, 2013, İstanbul, 2015, p. 201. 


\section{II - LA VIE PRIVÉE DANS L’ENTREPRISE}

C'est plus particulièrement au sein de l'entreprise où les devoirs d'obéissance et de discipline sont de rigueur, que le travailleur se voit confronté aux conséquences de certains de ses comportements (A).

Dans ce cadre, le contrôle de l'usage des moyens de communication par lesquels s'expriment les idées et opinions du salarié, présente un problème d'actualité que les tribunaux ont eu à résoudre (B).

\section{A - LES RELATIONS ENTRE COLlÈgUES AU TRAVAil}

On admet que la notion de «vie privée » ne s'arrête pas lorsque le travailleur pénètre dans l'établissement; la liberté et l'inviolabilité de cette vie devraient continuer d'être protégées même sur les lieux de travail.

Toutefois, il existe naturellement des limites : l'exposé des motifs de l'article 18 mentionne ainsi parmi les causes valables de licenciement le fait de « se mettre en relation avec d'autres personnes de manière à entraver le déroulement des services et à nuire au milieu du travail ».

Les exemples en ce domaine sont multiples et variés, allant des longs entretiens téléphoniques dont la fréquence et la longueur peuvent entraver le travail, jusqu'à des relations sentimentales entre collègues. Certes, sur ce dernier point, l'employeur ne devrait pas pouvoir apporter une interdiction, mais de telles interdictions existent bien dans la pratique dans certains règlements d'entreprise. La Cour de Cassation a dès lors précisé avec raison, suite à une rupture du contrat motivée par l'existence d'une relation sentimentale entre deux salariés, que « tant que la relation en question n'a eu d'incidence négative ni sur l'exercice du travail, ni sur l'ordre et la discipline de l'établissement, elle ne constitue point une juste cause de rupture ${ }^{19}$.

Ainsi, la vie privée du salarié ne peut en principe valablement justifier un licenciement, et une relation en dehors des heures de travail ne saurait constituer une cause de rupture.

Cependant, elle peut parfois avoir des incidences négatives sur le milieu de travail lorsque « le comportement du salarié qui a révélé à ses collègues sa relation avec une autre salariée, a mis cette personne dans une situation délicate. Dans un tel cas on ne peut normalement exiger que l'employeur maintienne en son établissement le salarié en question $»^{20}$.

La Cour de Cassation montre une certaine sévérité à l'encontre de relations extra-maritales dans le contexte du travail, et plus particulièrement entre supérieur et subordonné $(e)^{21}$. Ainsi la Cour a-t-elle considéré - mais pas à l'unanimité - comme une

19 9e Chambre civile, 25.2.1998, 365/2700, Kazanci.

$209^{e}$ Chambre civile, 8.11.2010, 35462/32188, Çil, p. 303.

$2122^{\text {e }}$ Chambre civile, 11.5.2015, 12601/16942, Legal, $n^{\circ} 49$, p. 444; $7^{\text {e }}$ Chambre civile, 9.2.2016,

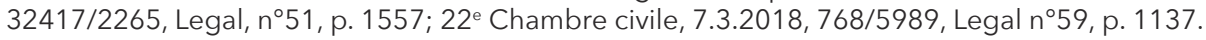


juste cause de licenciement (donc assimilée à une faute grave de l'article 25/II d) l'existence d'une relation sentimentale pourtant discrète entre un salarié marié et une collègue. La relation avait été révélée après que l'épouse se soit adressée à la direction, puis fut étayée par l'examen des courriels incriminés ${ }^{22}$. Il faut souligner en l'espèce que la problématique de ce type de preuve (captage de messages) n'avait été ni abordée, ni discutée dans ces décisions préalables.

Par ailleurs, les préférences sexuelles en tant que telles demeurent dans le domaine privé et ne peuvent constituer une cause de rupture ${ }^{23}$. Néanmoins, si une telle relation entre travailleurs d'un même établissement a provoqué des remous et polémiques sur le lieu de travail, le licenciement serait valide ${ }^{24}$. La doctrine souligne qu'il convient, à cet égard, de tenir compte du milieu de travail, du niveau d'instruction et des croyances des autres salariés ${ }^{25}$. Mais ici, ce qui importe n'est pas l'existence en soi de la relation ou de la préférence sexuelle, mais les effets concrets qui auraient entravé la bonne marche de l'établissement.

\section{B - L'USAgE DE L'INTERNET ET LE CAPTAGE DES MESSAgES}

L'usage à des fins personnelles du matériel, et surtout des moyens informatiques de l'entreprise, est un sujet d'actualité. Nombre d'arrêts se trouvent ainsi fondés sur des preuves qui apparaissent dans des correspondances via l'internet.

Alors se pose la question de savoir jusqu'où peut s'exercer le contrôle de la correspondance et la surveillance des agissements du salarié, ce dernier passant la plupart de son temps de vie dans l'entreprise ${ }^{26}$.

\section{1 - Le captage des messages}

Les ordinateurs, lignes internet et adresses de courriers électroniques fournis par l'entreprise, sont destinés à l'exécution du travail et ne peuvent en principe être utilisés à des fins personnelles. Certes, le salarié peut en faire usage dans des cas justifiables comme la nécessité ou l'urgence ${ }^{27}$. La Cour de Cassation admet aussi qu'une tolérance de fait, pendant une certaine durée (plus de six mois dans le cas examiné), pouvait être considérée comme un consentement tacite de l'employeur ${ }^{28}$.

$229^{e}$ Chambre Civile, 26.1.2001, 1999/18248, Günay, Şerh, p. 877.

$239^{e}$ Chambre civile, 19.3.2009, 36977/7401; 9e Chambre civile, 15.2.2016, 29678/2553, Keser, Sicil $2017, n^{\circ} 37$, p. $28 ; 9^{e}$ Chambre civile, 29.6.2016, 306/15491.

$249^{e}$ Chambre civile, 19.9.2005, 654/30275, Keser, Sicil 2017, n³7, p. 28.

25 H. Keser, «I Iş̧ç Davranışları Kapsamında İş Sözleşmesinin Özel Hayat, Aile Hayatı ve Cinsel Yönelimler Sebebi ile İşverence Feshedilmesi », Sicil iş Hukuku Dergisi, 2017, n³7, p. 9.

26 En Turquie la durée hebdomadaire du travail demeure de 45 heures, et le recours aux heures supplémentaires, bien que limité à 270 heures en une année, est très fréquent.

27 À ce sujet, voir notamment Z. Okur, İş Hukuku'nda Elektronik Gözetleme, İstanbul, 2011, p.148.

28 9e Chambre civile de la Cour de Cassation, 17.3.2008, 27583/5294, Kazanci. 
Quant au contrôle effectué, la jurisprudence a longtemps admis en Turquie que l'employeur pouvait exercer un contrôle sur les messages électroniques et autres moyens informatiques qu'il a lui-même fournis, contrairement à la doctrine qui met en avant la nécessité d'informer le personnel des limitations concernant leur usage, condition préalable pour justifier une rupture ${ }^{29}$.

De manière générale, la Cour de Cassation considère comme cause valable de rupture un usage abusif de l'ordinateur et de l'internet à des fins personnelles ${ }^{30}$. Un cas extrême s'était présenté lorsque l'ordinateur et l'adresse e-mail fournis par l'employeur, en vue de servir à la prestation de travail, avaient été utilisés à des fins non professionnelles, contenant de surcroît des propos injurieux à l'encontre du supérieur hiérarchique et dévoilant des secrets de l'entreprise.

Selon la Cour de Cassation, l'employeur a le droit de contrôler le contenu de la correspondance si l'ordinateur et l'adresse e-mail ont été fournis par ses soins, et l'usage à des fins personnelles, comme la teneur préjudiciable des messages, justifient une rupture pour faute grave selon l'article $25 /$ II b qui dispense du délai de préavis et du paiement d'indemnités de rupture ${ }^{31}$.

Or, pour que puisse s'exercer une intrusion dans ce courriel, la doctrine dominante pose comme condition la prise de connaissance et le consentement préalable exprès ou tacite du salarié. Les limitations concernant l'usage à des fins personnelles pourraient figurer dans le contrat de travail, la convention collective ou le règlement de l'entreprise ${ }^{32}$, et tout au moins un avertissement devrait être donné par la direction prévenant les salariés des restrictions portant sur l'usage personnel, ainsi que de l'éventualité d'un contrôle. Telle semble d'ailleurs avoir été la vue de la Cour Constitutionnelle en Turquie qui, en faisant largement référence à l'arrêt Barbulescu c. Roumanie de la Cour Européenne des Droits de l'Homme ${ }^{33}$, a motivé son arrêt en justifiant la rupture par l'existence de dispositifs d'un règlement d'entreprise qui restreignaient l'usage de l'internet et avertissaient les salariés de l'éventualité d'un contrôle ${ }^{34}$.

29 Sevimli, p. 203 ; H. Keser, İş̧i Davranışları Kapsamında İşçinin Talimatlara Aykııı Bilgisayar, İnternet ve Cep Telefonu Kullanımı Sebebi İle İş Sözleşmesinin İşverence Feshedilmesi, Legal IHSGHD, n62, 2019, p. 467. Voir également S. Öktem Songu, Isçilerin İsyerinde Özel Amaçlı İnternet ve E-Posta Kullanımına İsverenin Müdahalesi Üzerine Bir Değerlendirme, Prof. Dr. Sarper Süzek'e Armağan, p. 1057. Pour comparer voir Z. Okur, Iş Hukuku'nda Elektronik Gözetleme, İstanbul, 2011, p. 173: Selon l'auteur, le contrôle pourrait s'effectuer (seulement) dans les cas où l'usage personnel avait été interdit ou limité par la direction, et ceci dans la mesure des limitations imposées.

309 e Chambre civile, 9.5.2009, 36305/12393, et analyse par G. B. Yıldız, Sicil, Décembre 2009.

31 9e Chambre civile, 13.12.2010, 447/37516, Arrêt commenté et approuvé en l'espèce par F. Şahlanan, İşyerinde İşverence Sağlanan Bilgisayarı İşle İlgisi Olmayacak Şekilde Kullanımı - Haklı Fesih, Tekstil İşveren, Mars 2012, Karar İncelemeleri II, p. 103.

32 Şahlanan, p. 101.

33 Arrêt de la Cour européenne des Droits de l'Homme, Grande Chambre, 5 Septembre 2017, Requête $n^{\circ} 61496 / 08$.

34 Arrêt de la Cour Constitutionnelle, $2^{e}$ Section du 24.3.2016, Recours Ömür Kara et Onursal Özbek, n²013/4825, Journal Officiel du 19.5.2016, n²9708. 
Ainsi, la doctrine désapprouve et exclut une surveillance secrète exercée à l'insu du travailleur ${ }^{35}$. En l'absence de consentement, et excepté les cas où un intérêt supérieur (comme la sécurité ou salubrité de l'établissement) le justifierait, les preuves obtenues au moyen de courriers électroniques envoyés et reçus ne devraient pas être prises en compte ; l'inverse serait contraire au principe de respect de la vie privée.

Par ailleurs, l'article 189/2 du Code de Procédure civile dispose que « ne seront pas pris en compte des faits dont la preuve a été obtenue par des moyens illégaux ».

Telle a finalement été la décision de la Cour de Cassation, dans son arrêt récent du 7 août 201936: bien que le salarié ait en l'espèce effectivement fait un usage abusif et nuisible de l'internet, l'employeur ne peut pas se servir comme moyens de preuve des messages captés à son insu, sans distinction entre ce qui est privé et professionnel. La base légale de l'arrêt repose sur l'article 417 du Code des Obligations relatif au « devoir de protection et de respect de la personnalité du salarié, et de garantie d'un milieu de travail conforme au principe de bonne foi ».

La Cour souligne que la direction pouvait suivre et contrôler l'usage et le contenu des moyens informatiques mis à la disposition des salariés, mais qu'il était nécessaire d'en informer préalablement les intéressés.

\section{2 - L'expression d'opinions et teneur des messages}

Le salarié peut-il librement exprimer ses idées et opinions concernant la direction de son entreprise ? Une distinction est admise selon le poste occupé par la personne et la nature de l'entreprise ${ }^{37}$. Ainsi, une personne représentant l'entreprise et occupant une situation de confiance est tenue d'une certaine réserve.

Dans l'une des affaires examinées, un salarié en marketing avait adressé un courriel à un responsable de la direction, dans lequel il formulait des critiques assez irrévérencieuses sur sa manière de traiter certains clients. Dans la mesure où la teneur du message outrepassait les limites d'une simple critique, la Cour a considéré que cet agissement était de nature à créer des effets négatifs sur la bonne marche de l'entreprise, le qualifiant dès lors de cause valable de rupture ${ }^{38}$.

Un dernier cas bien différent, survenu dans un hôpital universitaire, fut celui d'un commentaire diffusé sur Facebook sous la photo d'une infirmière. L'auteur de la note - un gardien de sécurité de l'hôpital - avait exprimé un bref compliment, assez innocent. Ceci constitua néanmoins une cause valable de rupture, en raison des " effets négatifs sur le milieu du travail $»^{39}$.

35 Voir à ce sujet notamment : Öktem Songu, article précité, p.1057-1098; Keser, article précité, p. 445.

36 22e Chambre civile, 7.5.2019, 21857/9884, Çalışma ve Toplum 2020/1, nº4, p. 656.

37 Ertürk, p.124 ; E. Birben, İşçinin Özel Yaşamı Nedeniyle İ̧̧ Sözleşmesinin Feshi, İş Hukukunda Genç Yaklaşımlar II, Ed. T. Centel, İstanbul, 2016, p. 148.

38 9e Chambre civile de la Cour de Cassation, 23.10.2017, 2017/437-16298, Mess, 2018, n², p. 6.

$397 \mathrm{fe}$ Chambre civile de la Cour de Cassation, 10.3.2016, 2015/4359-2016/6048, Kazanci. 
Ce cas est un bel exemple de la prudence qu'il convient de montrer dans l'usage de ces moyens informatiques, dont la portée dépasse souvent les intentions de l'auteur et où un retour en arrière est quasiment impossible.

\section{Conclusion}

Le juge en Turquie prend bien en compte les droits de la personne au respect de la vie privée, tant dans l'appréciation des droits et obligations des parties que des causes de rupture du contrat de travail. La relative sévérité de la Cour de Cassation à l'égard de "relations inappropriées » se trouve confirmée par une jurisprudence constante, étayée par certains règlements d'entreprise déconseillant des relations non professionnelles entre salariés.

Or, en réalité, ce dont les entreprises se soucient n'est pas la morale, mais bien l'influence délétère que pourraient avoir sur le milieu du travail certains liens allant au-delà du strict niveau professionnel.

Dans le prolongement de la place majeure qu'a pris l'usage de l'internet, une évolution peut être observée d'une protection accrue du personnel contre le captage des courriels. Alors que, pendant longtemps, I'usage comme moyen de preuve de courriels obtenus par la direction était admis sans discuter de la légalité ou de la légitimité de leur usage devant les tribunaux, la Cour de Cassation semble désormais exiger que les personnes intéressées aient été préalablement prévenues que leur correspondance ainsi que l'usage des moyens informatiques, peuvent bien être suivis et contrôlés, puis utilisés contre eux. 